\title{
Simulating CXCR5 Dynamics in Complex Tissue Microenvironments
}

OPEN ACCESS

Edited by:

Peter Sims,

Columbia University, United States

Reviewed by:

Jamie Berta Spangler,

Johns Hopkins University,

United States

Irina Kufareva,

University of California, San Diego,

United States

*Correspondence:

Jason Cosgrove

jason.cosgrove47@gmail.com

Jon Timmis

jon.timmis@sunderland.ac.uk

Mark C. Coles

mark.coles2@kennedy.ox.ac.uk

Specialty section:

This article was submitted to

Systems Immunology,

a section of the journal

Frontiers in Immunology

Received: 30 April 2021

Accepted: 16 August 2021

Published: 07 September 2021

Citation:

Cosgrove J, Alden K, Stein JV

Coles MC and Timmis J (2021)

Simulating CXCR5 Dynamics in Complex Tissue Microenvironments.

Front. Immunol. 12:703088. doi: 10.3389/fimmu.2021.703088

\begin{abstract}
Jason Cosgrove ${ }^{1,2 *}$, Kieran Alden ${ }^{1}$, Jens V. Stein ${ }^{3}$, Mark C. Coles $^{4 *}$ and Jon Timmis ${ }^{5 *}$
${ }^{1}$ Department of Electronic Engineering, University of York, York, United Kingdom, ${ }^{2}$ Institut Curie, Université PSL, Sorbonne Université, CNRS UMR168, Laboratoire Physico Chimie Curie, Paris, France, ${ }^{3}$ Department of Oncology, Microbiology and Immunology, University of Fribourg, Fribourg, Switzerland, ${ }^{4}$ Kennedy Institute of Rheumatology at the University of Oxford, Oxford, United Kingdom, ${ }^{5}$ School of Computer Science, University of Sunderland, Sunderland, United Kingdom
\end{abstract}

To effectively navigate complex tissue microenvironments, immune cells sense molecular concentration gradients using G-protein coupled receptors. However, due to the complexity of receptor activity, and the multimodal nature of chemokine gradients in vivo, chemokine receptor activity in situ is poorly understood. To address this issue, we apply a modelling and simulation approach that permits analysis of the spatiotemporal dynamics of CXCR5 expression within an in silico B-follicle with single-cell resolution. Using this approach, we show that that in silico B-cell scanning is robust to changes in receptor numbers and changes in individual kinetic rates of receptor activity, but sensitive to global perturbations where multiple parameters are altered simultaneously. Through multi-objective optimization analysis we find that the rapid modulation of CXCR5 activity through receptor binding, desensitization and recycling is required for optimal antigen scanning rates. From these analyses we predict that chemokine receptor signaling dynamics regulate migration in complex tissue microenvironments to a greater extent than the total numbers of receptors on the cell surface.

Keywords: B cells, chemokines, systems biology, G-protein coupled receptors, mathematical modelling

\section{INTRODUCTION}

Through interactions with non-hematopoietic stromal cells, B-cells generate tightly compartmentalized structures known as B-cell follicles within secondary lymphoid tissues. The follicular niche is responsible for coordinating B-cell homeostasis, activation and affinity maturation; precisely modulating B-cell activity through a temporal sequence of site-specific cues (1). The precise spatial positioning of B cells within the follicular niche is regulated by G-protein coupled receptors (GPCRs) that bind signaling lipids and chemokines inducing directed migration along a concentration gradient $(2,3)$. In this study we focus on CXCR5 the cognate receptor for CXCL13, a chemokine produced by stromal fibroblasts that is essential for follicle formation and maintenance (4-6). CXCR5 deficient B cells display aberrant migratory behaviors within lymph nodes, a phenotype associated with impaired homing to follicles $(4,7)$. CXCR5-/- B cells injected into a WT host are known to have different migration characteristics within LN follicles in comparison to WT B cells (8). Western blotting of pooled lymph nodes, in vitro migration assays, and measurements of the dissociation constant of CXCR5-CXCL13 suggest that the follicular 
concentration of CXCL13 ranges from 10-50nM. Studies of virally challenged lymph nodes however suggest that CXCR5mediated migration can occur even when CXCL13 mRNA levels drop by $95 \%$ (8); this is consistent with studies that suggest a difference of 10 signaling receptors across a cell is sufficient to induce chemotaxis along a gradient $(9,10)$.

GPCRs are controlled by a complex and dynamic regulatory network that spans broad spatiotemporal scales, allowing cells to detect subtle asymmetries in chemokine concentrations (2, 10, 11). In terms of temporal sensing mechanisms, it has been reported that stable gradients of the homeostatic chemokines CCL19 and CXCL12 fail to promote the persistent directed migration of dendritic cells or neutrophils in vitro. Strikingly, rising chemokine concentrations were required to induce prolonged chemotaxis, in a mechanism dependent on GPCR kinase mediated negative regulation of receptor signaling (12). However, it is still unclear to what extent CXCR5 shapes gradient sensing within the primary B-follicle.

Despite recent advances in super-resolution imaging that permit single molecule analysis of GPCR dynamics in vitro (13), it is not yet possible to track CXCR5 dynamics in situ. To address this limitation many mathematical models have been developed to assess molecular dynamics through simulation analyses. The dynamics of CXCR4 and CXCR5 expression within the germinal center (GC) have been assessed using ordinary differential equations, an approach that predicts changes to components over one independent variable (e.g. time) on a continuous scale (14). Analysis of this model highlighted the importance of receptor down-regulation at the cell surface as a cell-intrinsic mechanism to govern migration patterns, predicting an oscillatory pattern of CXCR5 expression as B-cells migrated between the light and dark zones of the germinal center. Agent-based modeling, a technique that focuses on modeling the aggregate behaviors of individual cells or "agents" has also been employed to study this phenomenon. An $\mathrm{ABM}$ approach has been used to demonstrate that receptor internalization is a source of instability during tissue formation, leading to structural changes in tissue architecture (15) and that chemokine sensitivity is quickly down-regulated within the GC (16). Based on these experimental and theoretical studies, we hypothesize that receptor dynamics contribute to B-cell homeostasis by temporally regulating gradient sensing. However, much of the theoretical work that has been performed to date assume idealized chemokine gradients, and did not consider the complexity of tissue architecture, or the multimodal nature of CXCL13 diffusion (17). It is thus unclear if these models can accurately describe CXCR5 dynamics in situ.

To address our hypothesis that receptor dynamics contribute to $\mathrm{B}$-cell homeostasis by temporally regulating gradient sensing we use the CXCL13Sim multiscale modeling platform (17) to assess the sensitivity of $\mathrm{B}$ cell scanning to perturbations in CXCR5 in silico. In previous work we have used CXCL13Sim to map the spatial distribution and dynamics of CXCL13 within lymph nodes. In this work we use the CXCL13Sim platform to ask how receptor dynamics regulate cell migration within complex tissue microenvironments. This novel system builds upon previous theoretical work on receptor dynamics by incorporating the 3 -dimensional geometry of CXCL13 ${ }^{+}$ follicular stromal networks, and the multimodal mobility of CXCL13 within the follicle.

Specifically, we apply parameter robustness to demonstrate that in silico B-cell scanning is robust to changes in receptor numbers and changes in individual kinetic rates of receptor activity, but sensitive to global perturbations where multiple parameters are altered simultaneously. This result is corroborated by a multiobjective optimization analysis to identify the configuration of CXCR5 signaling that maximizes the rate of antigen scanning. This analysis showed that for optimal scanning rates to occur multiple parameters regulating the dynamics of CXCR5 expression and signaling need to be tightly modulated. In addition, we perform single cell tracking to determine spatial and temporal patterns of expression in response to the complex gradients that occur in vivo. Taken in concert, our results suggest that the rapid modulation of chemokine receptor activity permits efficient antigen scanning by B-cells. This result has an important consequence for our understanding of B-cell migration in the context of complex tissue microenvironments which may benefit from targeting receptor dynamics, as opposed to downregulating total numbers of receptors.

\section{MATERIALS AND METHODS}

\section{Overview of the Development Framework}

To ensure a principled and transparent design process we employ the CoSMoS (Complex System Modelling and Simulation) process, a framework to guide the modelling and analysis of complex systems (18). The design and implementation decisions made when constructing a simulator are influenced by the overarching scientific objectives of the work, with simulation results interpreted in this context $(19,20)$. To argue that the simulator fulfils its remit, acceptance tests, key design decisions, and information used to inform the design, development and validation of the model and simulation are presented as arguments over evidence using a visual notation derived from goal structuring notation (19). The argumentation structures are provided in files S3-S4 and can be opened using the ARTOO tool (http://artoofree.simomics.com/).

\section{Software Development and Computer Infrastructure}

Within the simulation platform each module was developed using Java and the MASON ${ }^{1}$ ABM library version 19 (21) in an iterative process of implementation, validation and refactoring using Acceptance Test-Driven Development (ATDD) (22). Tests are continually assessed and refined as the project progresses and are incorporated into an automated regression framework using the java library JUnit ${ }^{2}$ with test coverage quantified using the eclipse plugin eclEmma ${ }^{3}$. Simulations were executed on a Linux

\footnotetext{
${ }^{1}$ Available from http://cs.gmu.edu/ eclab/projects/mason.

${ }^{2}$ Available from http://junit.org/junit $4 /$.

${ }^{3}$ Available from http://www.eclemma.org/download.html.
} 
Cluster made up of 64 CPU 256GB memory nodes and running Fedora 22 and an Oracle grid engine.

\section{Model Parameters, Parameter Ranges, and Outputs \\ Quantification of pLN Follicle Size and Cellularity}

Follicle volume was obtained from two publications $(23,24)$. Kumar et al. determined the mean volume of a popliteal LN follicle using OPT scanning on BABB-immersed pLNs in conjunction with software based $3 \mathrm{D}$ reconstruction and quantification. Their analysis shows that typical pLN volume is $1.25 \mathrm{~mm}^{3}$, dividing that by $10 \%$ (the percentage of volume accounted for by follicles) gives a volume of $1.25 \times 10^{8} \mu \mathrm{m}^{3}$ for all of the B-zones in one LN and dividing that by the number of follicles (18 [7-35]) gives $6.94 \times 10^{6} \mu^{3}$. In addition the authors observed a strong correlation $\left(\mathrm{r}^{2}=0.93\right)$ between the size of the lymph node and the number of follicles. Values obtained by Irla et al. of inguinal lymph nodes suggest that total $\mathrm{LN}$ volume is $2.4 \mathrm{~mm}^{3}$. Dividing this by $18 \%$ gives the $\%$ follicular volume. We then divide this value by the number of follicles (12.5) to get the volume of one follicle $\left(3.46 \times 10^{7} \mu \mathrm{m}^{3}\right)$. The discrepancy between the two sets of measurements can most likely be attributed to differences in lymph node morphology at different anatomical sites and different experimental procedures.

B-cell density was determined by quantifying $\mathrm{CD} 9^{+}$cells using flow cytometry in conjunction with Accucheck counting beads ( $\mathrm{n}=5$ mice, 3 separate experiments) and averaging total pLN counts by the mean number of follicles per lymph node. Stromal cell densities within a fixed follicular volume were determined from the number of nodes obtained from a $400 \mu \mathrm{m}$ x $400 \mu \mathrm{m}$ x $30 \mu \mathrm{m}$ image (Table 1) of a follicle obtained from a Cxcl13-EYFP reporter mouse.

From this data we define a popliteal lymph node as having a volume of $1.25 \mathrm{~mm}^{3}, 15 \%$ of which is defined as a B-zone split between 15 follicles. Within this lymph node each follicle is spheroidal with a total volume of $1.25 \times 10^{7} \mu \mathrm{m}^{3}(\sim 250 \times 250 \mathrm{x}$ $350 \mu \mathrm{m})$ and contains $4.8 \times 10^{4} \mathrm{CD} 9^{+} \mathrm{B}$ cells.

\section{B-Cell Migration}

The migration patterns of $\mathrm{B}$ cells were measured by injecting $5 \mathrm{x}$ $10^{6}$ purified B cells from C57BL/6 or CXCR5 deficient mice labelled with the fluorescent cell staining dyes $(((4-$ chloromethyl)benzoyl)amino)tetramethylrhodamine) CMTMR into age- and sex- matched wild type mice and imaged with twophoton microscopy (8). This data was provided by the Stein lab. WT B cells have a velocity (expressed as median[lower quartile upper quartile]) of 8.0 [2.3 - 10.3] $\mu^{2} \mathrm{~min}^{-1}$, a meandering index of $1.1[0.1-2.4]$ and a motility coefficient of 15.6 [0.1 96.7] $\mu \mathrm{m}^{2} \min ^{-1}$.

\section{CXCR5 Expression}

The number of CXCR5 molecules per naïve B-cells in lymph nodes is unknown, however data from similar systems suggests that the amount of receptors is in the range of $10,000-100,000$ receptors $(31,32)$.

\section{CXCL13 Diffusion Constant}

A value of $7.6 \pm 1.0 \mu \mathrm{m}^{2} \mathrm{~s}^{-1}$ was obtained using high-speed singlemolecule imaging. An upper bound for this value was determined using the Einstein stokes relation, assuming a Stokes radius of $3.48 \mathrm{~nm}$ and that the molecule undergoes free diffusion in water $\left(146 \mu \mathrm{m}^{2} \mathrm{~s}^{-1}\right)$.

\section{Kd and CXCL13 Secretion Rate}

The total amount of chemokine from pooled lymph nodes (33), in vitro migration assays (5), and ligand binding constants (34) were used to derive upper and lower limits of a likelihood distribution for CXCL13 concentrations in vivo. Taken in concert these analyses suggest that the value lies in the range 1 - 50nM with a baseline follicle concentration assumed to be $10 \mathrm{nM}$ that we set as our binding affinity $\mathrm{Kd}$. This is consistent with expected ranges of Kd for CCR7 and CCL19 where similar concentrations have been described to sufficiently trigger downstream signalling of CCR7 following binding of CCL19 in G-protein loading assays (35), downstream signalling assays (36) and microfluidic migration assays (37).

\section{$\mathrm{Ki}$ and $\mathrm{Kr}$}

The two rate constants $\mathrm{Ki}$ and $\mathrm{Kr}$ associated with receptor internalization and recycling were estimated from experimental data on receptor desensitization and resensitization neutrophils and from mathematical modelling of B-cell migration $(31,32)$.

\section{CXCL13 Decay Rate}

Chemokine's are further regulated in vivo by proteases providing rapid enzymatic modulation of bioactivity and availability. Systematic analyses of proteomic half-lives suggests a broad range of possible values, which we constrain between 15 minutes to 48 hours $(38,39)$. Assuming a constant decay rate this yields rates between 0.015 and $0.0002 \mathrm{~s}^{-1}(40)$.

\section{Calibration to Establish Baseline Simulation Behaviors}

To constrain each parameter value, lower and upper bounds were set on the basis of direct experimental measures or derived from indirect evidence from the wider literature. Parameters were systematically changed and compared to experimental datasets using the non-parametric Mann-Whitney U-Test given outputs were not normally distributed, as determined by a Shapiro-Wilk test for normality (41). To assess the robustness of our baseline calibrated parameter values, outputs from best-fit parameter sets were compared to gene knockout experiments. Parameter sets that failed to reproduce statistically comparable results to both wild type and gene deficient mice were omitted from any further analyses.

\section{Quantification of Model Uncertainty}

To quantify sources of uncertainty in the our simulator we used the R software package SPARTAN (42). This package contains a 
TABLE 1 | Summary of model outputs.

\begin{tabular}{|c|c|}
\hline Model Output & Description \\
\hline Total Displacement & $\begin{array}{l}\text { Record the steps taken by cells and calculate displacement over a fixed } \\
\text { time period using vector addition. }\end{array}$ \\
\hline Net Displacement & Euclidean distance between the first and last position of the cell \\
\hline Cell Velocity & Total displacement/time \\
\hline Motility Coefficient & Net displacement ${ }^{2 /} 6^{\star}$ time \\
\hline Meandering Index & $\sqrt{\text { Time }}{ }^{*}$ (net displacement/total displacement) \\
\hline Scanning Rate & Number of unique gridspaces reached within a single simulation run \\
\hline CXCR5 state & A vector containing the number of free, ligand-bound, desensitized, and $i$ \\
\hline
\end{tabular}

Following each individual simulation run, the following metrics are calculated for each B-cell agent.

suite of statistical techniques (described in more detail in the following sections) specifically designed to help understand the relationship between the simulator and the physical system it describes.

\section{Quantification of Aleatory Uncertainty}

CXCL13Sim is non-deterministic and therefore can produce different outputs under the same parameter inputs. To determine how many runs are required to give a representative output for a given parameter set we perform an aleatory analysis. To quantify aleatory uncertainty, 20 distributions were generated and contrasted for each sample size. A distribution of median responses for each simulation run is generated for each of the 20 subsets. Distributions 2-20 are contrasted with the distribution from the 1st set using the Vargha-Delaney A-Test (43), a non-parametric effect magnitude test that establishes scientific significance by contrasting two populations of samples and returning the probability that a randomly selected sample from one population will be larger than a randomly selected sample from the other. Values of 0.5 indicate that the medians are the same while values of 1 and 0 mean that there is no overlap. In our analyses we set thresholds for small (0.56), medium (0.66) and large (0.71) effect sizes based on values suggested by $(44,45)$ and define a significant behavioral alteration as one where the A-test statistic exceeds the medium threshold.

\section{Local and Global Parameter Robustness Analyses}

To quantify how sensitive simulation outputs are to perturbations in parameters we applied a number of sensitivity analysis (SA) techniques. For local analyses each parameter is adjusted, with all other parameters remaining at their calibrated value. The Vargha-Delaney A-Test described previously implemented in SPARTAN is employed to determine if changing the parameter value has led to a significant behavioral alteration in contrast to the baseline simulation. We define a significant behavioral alteration as one that surpasses a threshold A-test value of 0.66

To perform a global sensitivity analysis, we use two parameter sampling techniques, LHC (Latin-Hypercube) and eFAST (Extended Fourier Amplitude Sampling Test). Through Latin hypercube sampling, values for each parameter are selected with the aim of ensuring efficient coverage of the parameter space. Parameters that have significant impact on simulation behaviors are identified through calculation of a Partial Rank Correlation Coefficient (PRCC), a robust measure for quantifying non-linear relationships between an input and output (46). To calculate the PRCC the data are rank-transformed, and for each parameter, two linear regression models are found, the first representing the input parameter in terms of the other parameters and the second represents the output measures in terms of the other parameters. A Pearson correlation coefficient for the residuals from those two regression models gives the PRCC value for that specific parameter. Thus, PRCCs characterize a linear relationship between input $\mathrm{x}$ and output $\mathrm{y}$ after the linear effects of the other inputs on $y$ have been discounted. A significance test is performed to assess if a PRCC is significantly different from zero (46).

Latin Hypercube Sampling (LHS) and Partial Rank Correlation Coefficients (PRCC) identify parameters that have a significant effect on model outputs. This approach facilitates an understanding of what parameters should be targeted to achieve a desired response but does not indicate which parameter uncertainties have the greatest impact on output variability. The extended Fourier Amplitude Sampling Test (eFAST) is a variance decomposition method that can be used to address this issue $(47,48)$. In this approach input parameters are varied, causing variation in model output. The algorithm then partitions the output variance, determining what fraction of the variance can be explained by variation in each input parameter. In this scheme, parameters values are sampled using a sinusoidal function of a particular frequency. Each parameter is taken in turn and sampled at a frequency that is much larger than the other parameters. Due to the symmetry of a sinusoidal function it is possible to choose the same parameter set more than once, therefore a re-sampling scheme in which a phase shift is introduced at each frequency is encouraged (46). Through Fourier analysis using these frequencies, variation in output can be partitioned between the parameters, giving an indication of the impact each has on simulation response. This process is repeated for an extra parameter, the 'dummy' parameter that has an arbitrary value range but no impact on simulation behavior. This enables a comparison between the impact of each parameter and one known to have no effect on simulation response. To quantify the influence of each parameter, two sensitivity indexes are calculated for each parameter-response pairing: a first-order (Si) and total order sensitivity (STi) index. The first indicates 
the fraction of output variance in that response that can be explained by the value assigned to the parameter. The latter indicates the variance in that response caused by higher-order non-linear effects between the parameter and the others under investigation. To determine the significance of these metrics, indexes are compared to those obtained for the 'dummy' parameter using a two-sample t-test.

\section{Simulation Emulation With Machine Learning}

The training dataset for emulator development was obtained using Latin hypercube sampling, with 1000 parameter sets. Each set was executed 100 times to mitigate aleatory uncertainty, and median responses calculated to summarize simulator performance under those conditions. The data set was partitioned into training (75\%), testing (15\%) and validation (10\%) datasets.

The supervised learning approach used to generate CXCL13emulator was an artificial neural network, an approach inspired by the neuronal circuits in the brain, with computations structured in terms of an interconnected group of artificial neurons. During the learning phase, the weighting of connections between neurons are adjusted in such a way that the network can convert a set of inputs (simulation parameters) into a set of desired outputs (simulation responses). The ANN-based emulator was developed in the $\mathrm{R}$ package SPARTAN with supervised learning of the data achieved through backpropagation of errors. To determine optimal hyperparameters of the network we performed ten-fold cross validation on a selection of structures with thirteen inputs (the parameters) and four outputs (speed, meandering index, motility coefficient and checkpoints reached), with one to four hidden layers. The accuracy of each fold was determined to be the mean squared error between the predicted cell behavior responses and those obtained by the simulator, and the accuracy of the network structure determined to be the average of these ten-fold root mean squared errors.

\section{Multi-Objective Optimization}

Multi-objective optimization of the CXCL13emulator was performed using the non-dominated sorting genetic algorithm II (NSGA-II), a multi-objective genetic algorithm (49). This analysis was performed in $\mathrm{R}$ using the package mco v15.0 ${ }^{4}$. The four objectives to be assessed by the algorithm were to: minimize the root mean squared error between emulator and simulator responses for cell speed, meandering index and motility coefficient; and maximize scanning rates. Values for generation number, mutation and crossover probabilities were determined by a global sensitivity analysis whereby values for mutation and crossover rates were sampled between 0.1 and 1.0 (intervals of 0.1 ) and values for the number of generations was sampled between 200 and 500 (intervals of 100). We chose parameters that performed well on all three objectives and maximized the variance of the parameter inputs.

\footnotetext{
$\overline{{ }^{4} \text { Available from https://CRAN.R-project.org/package }=\text { mco. }}$
}

\section{RESULTS}

CXCL13Sim is a 3D hybrid multiscale model developed using the CoSMoS (Complex System Modelling and Simulation) process, a framework to guide the modelling and analysis of complex systems. In this scheme B cells are modelled as agents that adjust their behaviors with respect to vector and ordinary differential equationbased calculations adapted from a published scheme (50) that explicitly accounts for gradient detection and the dynamics of GPCR expression on the cell surface (Figures 1A, B). B-cell agents respond to CXCL13 gradients generated by in silico stromal cell networks. In silico stroma are modeled as a graph of nodes and edges (Module 1) and secrete CXCL13, which is represented by a double precision floating point number on a discretized grid with diffusion modeled using a discretized partial differential equation. A full overview of model parameters and outputs is provided in Tables 1 and 2). Within this scheme we find that the migration characteristics of in silico B-cells are consistent with those obtained by multiphoton imaging of both wild-type and $\mathrm{CXCR} 5^{-/-} \mathrm{B}$ cells (Figure S1A) and that the topological parameters of the CXCL13 ${ }^{+}$ follicular reticular network are consistent with those measured in vivo (Figures S1B, C). A full description of the design, implementation, and validation of CXCL13Sim, along with parametric and aleatory uncertainty quantification analyses, is provided in file S2. In Files S3-S4 we provide an argumentation structure detailing all of the key assumptions of our model and links to the data that supports our assumptions.

To quantify parametric uncertainty in our simulation platform we apply sensitivity analysis (SA) techniques, which broadly speaking can be split into two categories: local and global. Local analysis techniques examine how robust the simulation is to a perturbation of a single parameter value (herein referred to as a One At a Time (OAT) analysis). However, local SA techniques cannot reveal compound effects where one parameter's influence is dependent on the value of another. Such effects may be elucidated using global analysis techniques that perturb multiple parameters simultaneously.

To assess the consequences of CXCR5 deficiency on the induction of humoral immune responses we quantified B-cell trafficking of $\mathrm{CXCR}^{-/-} \mathrm{B}$ cells in silico and find a significant reduction in scanning rates (the number of unique gridspaces reached by an in-silico B-cell within a single simulation run) compared to wild-type B cells (Figure 1C). While complete loss of the receptor yielded a strong phenotype, perturbations to total numbers of CXCR5 across an order of magnitude (between 10100,000 receptors) led to modest changes in the baseline rate of network scanning (Figure 1D). The distribution of scanning rates in this OAT analysis (Figure 1D), were far narrower than the distributions of scanning rates observed when performing a global multiparametric perturbation analysis (Figure 1E). This yielded the hypothesis that the dynamics of CXCR5 signalling modulate antigen scanning to a greater extent than total receptor numbers.

To assess this hypothesis we performed a suite of parameter sensitivity analyses, using the Spartan analysis software package in $\mathrm{R}$ (42). Using this approach, we find that overall scanning rates were robust to one-at-a-time perturbation to both $\mathrm{K}_{\mathrm{on}}$ and $\mathrm{K}_{\text {off }}$ 
A

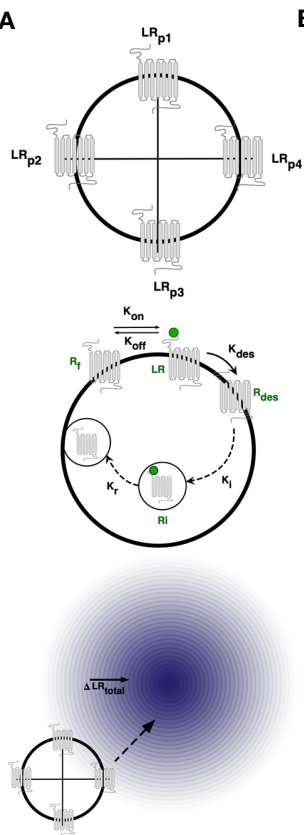

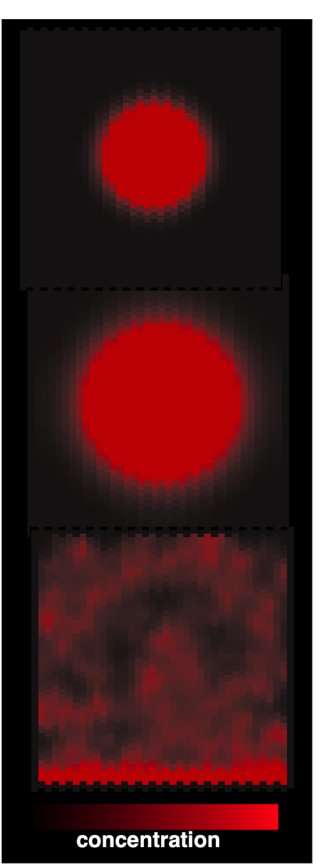

C

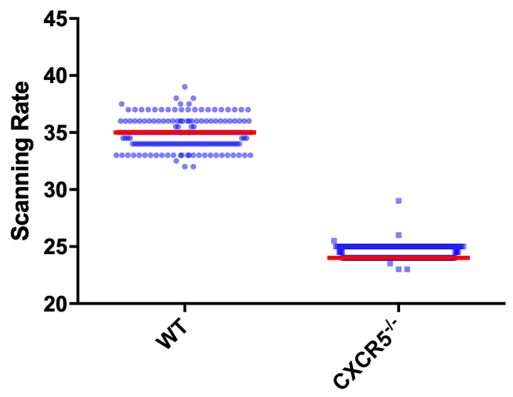

E

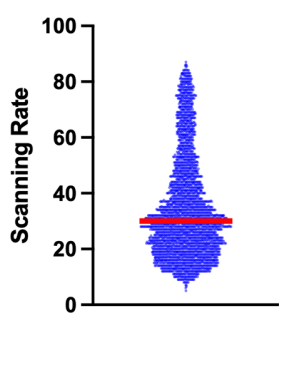

D

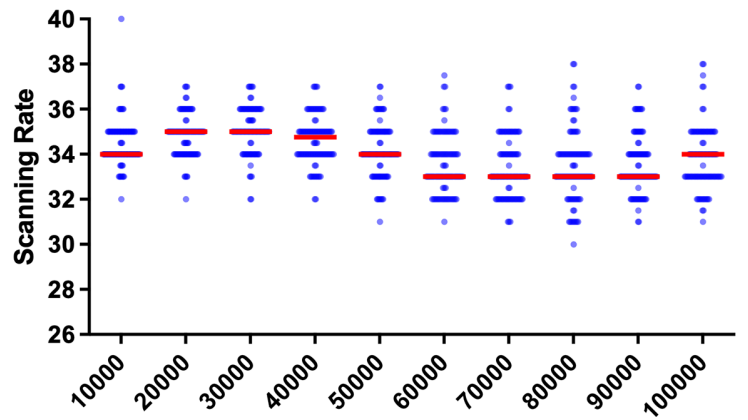

FIGURE 1 | In silico CXCR5 modelling: Graphical Overview of the gradient sensing algorithm (A), including receptor internalization and recycling (B) in the context of CXCL13 gradients (C) Quantification of scanning rates in wild-type and CXCR5 deficient B cells. (D) Quantifying scanning rates when perturbing total receptor numbers holding all other parameters fixed at baseline values. Median values are shown with error bars representing the I.Q.R. (E) Distributions of scanning rates observed from our global parameter perturbation analysis.

TABLE 2 | Summary of parameter values.

\begin{tabular}{|c|c|c|c|c|}
\hline Parameter & Value & Unit & Range & Reference \\
\hline Total Number of B cells & 6000 & cells & Constant & Measured \\
\hline Total Number of FDCs & $\sim 200$ & cells & Constant & Measured \\
\hline Total Number of BRCs & $\sim 450$ & cells & Constant & Measured \\
\hline Proportion of Cognate Cells & 5 & $\%$ & Constant & - \\
\hline Displacement constant & 7.4 & $\mu \mathrm{m} \min ^{-1}$ & {$[1-10]$} & Calibrated \\
\hline Total receptor number & 48,000 & Receptors & $\begin{array}{l}{[10,000-} \\
100,000]\end{array}$ & (11) \\
\hline$K_{\text {on }}$ & $\begin{array}{l}4.8 \times \\
10^{5}\end{array}$ & $\mathrm{Ms}^{-1}$ & {$\left[1 \times 10^{5}-1 \times 10^{6}\right]$} & (26) \\
\hline $\mathbf{K}_{\mathbf{i}}$ & 0.0033 & $s^{-1}$ & {$[0.001-0.01]$} & $(11,27)$ \\
\hline $\mathbf{K}_{\mathrm{des}}$ & 0.075 & $\mathrm{~s}^{-1}$ & {$[0.01-0.1]$} & $(11,27)$ \\
\hline CXCL13 decay rate & 0.007 & $\mathrm{~s}^{-1}$ & {$[0.0002-0.05]$} & $(29,30)$ \\
\hline CXCL13 diffusion rate & 7.6 & $\mu \mathrm{m}^{2} \mathrm{~s}^{-1}$ & {$[0-146]$} & Measured \\
\hline$\alpha 1$ (measure of cell persistence during chemotaxis where lower values indicate higher polarity) & 0.475 & - & $0-1$ & Calibrated \\
\hline $\begin{array}{l}\alpha 2 \text { (measure of cell persistence during random migration where lower values indicate higher } \\
\text { polarity) }\end{array}$ & 3.8 & - & Constant & Calibrated \\
\hline
\end{tabular}

For each parameter the name, baseline value and range used for uncertainty and sensitivity analyses is provided. Parameter values were determined experimentally or in cases where no direct experimental value exists, upper and lower limits were derived from indirect evidence, baseline values were then determined by fitting the model to experimental datasets (calibration). The model was further validated against migration data from CXCR5 ${ }^{-1}$ B cells and parameters were removed where possible. The values for stromal cells are averaged over 250 runs with individual values varying to a small extent between runs due to stochastic network formation. 
rates. This suggests that scanning is robust to the affinity of binding (defined as $1 / \mathrm{K}_{\mathrm{d}}$ or $\mathrm{K}_{\mathrm{on}} / \mathrm{K}_{\text {off. }}$ ) over the ranges examined, and that efficient scanning can occur over a broad range of CXCL13 concentrations (Figure 2A). OAT perturbations to desensitization, $K_{i}$ and $K_{r}$ rates led to modest changes in scanning rates but global sensitivity analyses suggest complex interactions between these parameters, and that combinatorial perturbations may yield a synergistic effect (Figures 2B, C). Thus, using local and global sensitivity analyses we find that dynamic modulation of signaling has a greater impact on scanning rates than overall receptor numbers. To further explore this prediction, we perform multi-objective
A

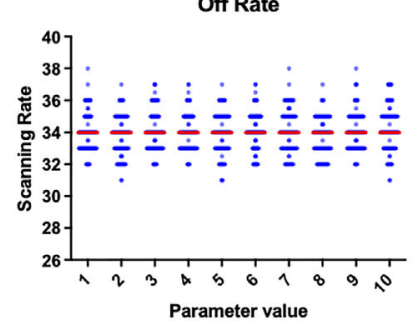

Internalisation Rate

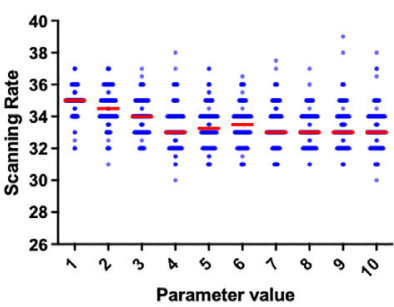

Desensitisation Rate

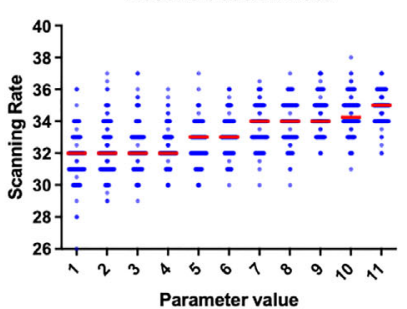

Affinity Constant

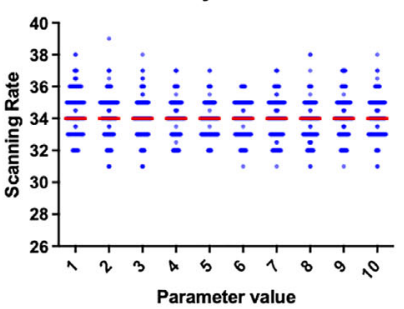

Recycling Rate

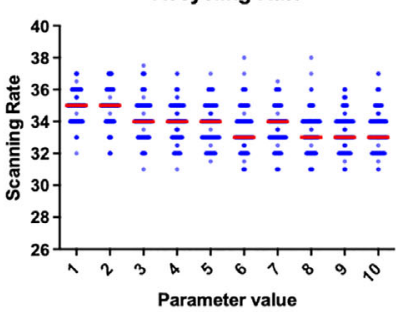

B

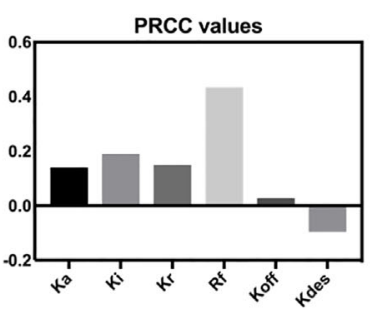

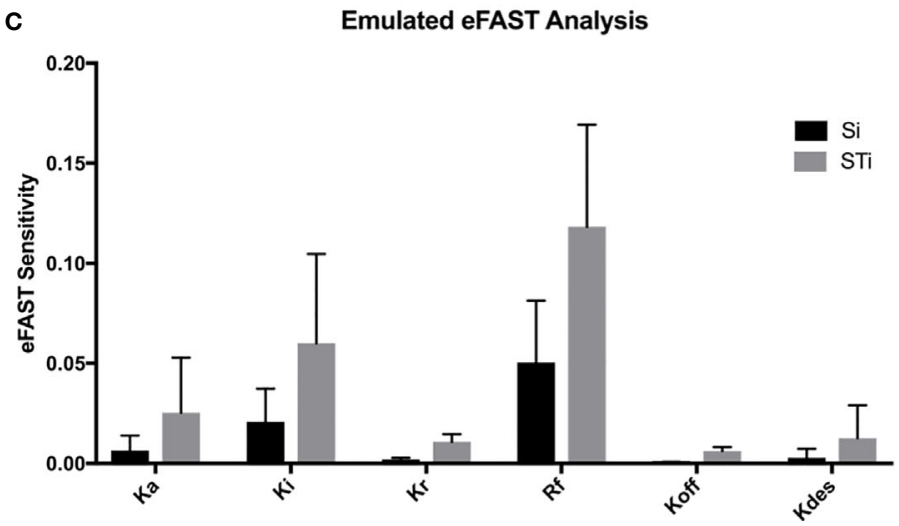

FIGURE 2 | OAT perturbation of kinetic parameters that regulate CXCR5 signaling. (A) Perturbing kinetic parameters regulating CXCR5 activity and assessing the effect on in silico scanning rates. (B) Partial rank correlation coefficients quantifying the sensitivity of in silico scanning rates to perturbations in a given parameter, taking the influence of the other parameters into account. (C) eFAST analysis of parameter sensitivities using the CXCL13emulator Si (black) represents the fraction of output variance that can be explained by the value assigned to that parameter. STi (grey) represents the variance caused by higher order non-linear effects between that parameter and others explored. Bars represent the mean value for either Si or STi, with error bars representing the standard error over three resample curves. 
optimization (MOO) of the CXCL13emulator. To achieve this, we employ a specialized class of optimization algorithms known as multi-objective evolutionary algorithms (MOEAs) to determine what CXCR5 configurations maximize B-cell scanning rates. MOEAs use a similar principle to the germinal center (GC), using mutation and selection to determine a set of solutions (a Pareto front) where improvement in one objective cannot be obtained without compromising performance for another objective. In the GC this trade-off occurs because a mutation that improves binding for one epitope on a complex microbial antigen may reduce binding to other epitopes, reducing overall binding avidity. This GC-inspired analysis shows that our objectives are conflicting, with increased scanning rates leading to poorer agreement between emergent cell behaviors in silico and laboratory measures (Figure $\mathbf{3 A}$ ). Analysis of the parameter distributions corresponding to the population of Pareto optimal solutions shows that $\mathrm{R}_{\text {total }}, \mathrm{K}_{\mathrm{on}}$, $\mathrm{K}_{\text {des }}, \mathrm{K}_{\mathrm{i}}$ and $\mathrm{K}_{\mathrm{r}}$ are highly skewed towards high values while $\mathrm{K}_{\text {off }}$ is skewed towards lower values, consistent with the hypothesis that dynamic modulation of signaling through rapid receptor turnover and desensitization promotes effective migration (Figure 3B).

Taken in concert, our data suggest that modulation of receptor activity is a key determinant of trafficking behaviors. Leveraging recent insights into CXCL13 gradient formation (17), we use simulation analysis to resolve spatial and temporal components of the CXCL13-CXCR5 regulatory axis. To assess cell specific contributions to follicular scanning rates we performed histological analysis of lymph node tissue sections from Cxcl13-Cre/Tdtomato R26R-EYFP (abbreviated as Cxcl13EYFP) mice (51). In Cxcl13-EYFP mice, EYFP acts as a lineage marker, endogenously expressed in cells that originate from a CXCL13-producing precursor, while TdTomato expression (red fluorescent protein, RFP) is confined to cells with current CXCL13 promoter activity. Within the $\mathrm{CXCL} 3^{+}$stromal cell populations we define 3 subsets: CXCL13 ${ }^{+} \mathrm{CD} 21 / 35^{+}$Follicular Dendritic Cells (FDCs) and CXCL13 ${ }^{+} \mathrm{CD} 21 / 35^{-}$reticular cells (CD21 ${ }^{-}$RCs) comprising reticular cells located underneath the subcapsular sinus (marginal reticular cells - MRCs), and at the outer follicle (B-zone reticular cells - BRCs) (17). Using the relative TdTomato intensities as a proxy for CXCL13 secretion we infer distinct secretion rates for each stromal cell subset. Using this secretion profile, we find no significant change in overall scanning rates (Figures 4A, B) but did find that B-cells localize at the subcapsular sinus, a key site for antigen presentation (Figure 4D). To further assess how relative secretion rates of stromal cells can affect scanning rates we performed a sensitivity analysis and find that overall scanning rates are highest with low rates of secretion from reticular cells, while for FDCs highest rates were found at intermediate secretion rates (Figure $4 \mathrm{C}$ ). We then followed the temporal (Figure 4E) and spatial (Figure 4F) dynamics of CXCR5 expression on individual B-cell agents. Analysis of signaling and free receptors on the cell surface shows a dynamic pattern of expression (Figure 4E). Analysis of each receptor subset shows that desensitized receptors were the highest CXCR5 subset followed by internalized receptors and relatively few free

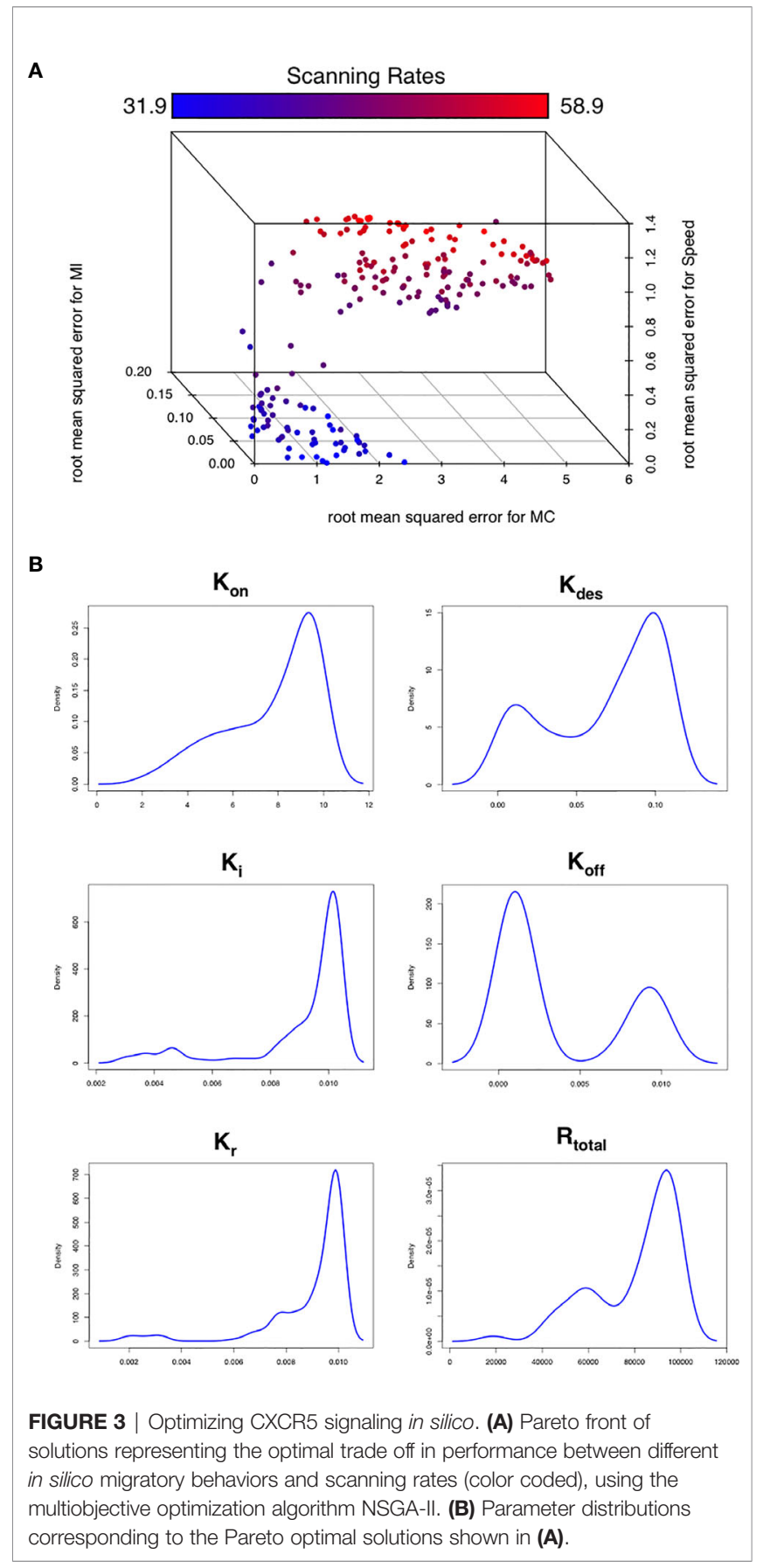

and signalling receptors on the cell surface (Figure 4F). Interestingly, CXCR5 expression was spatially regulated with highest levels of signalling occurring at the subcapsular sinus, a profile associated with low numbers of free receptors and high numbers of internalized receptors (Figure 4F).

\section{DISCUSSION}

CXCR5 is a key molecular player in CXCL13-mediated cross talk between B cells and stromal cells. In vitro studies of GPCR- 
A

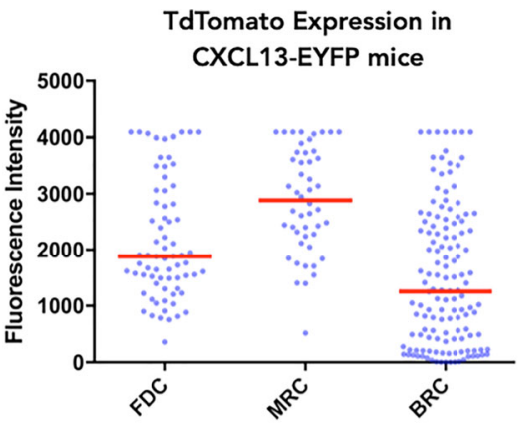

C

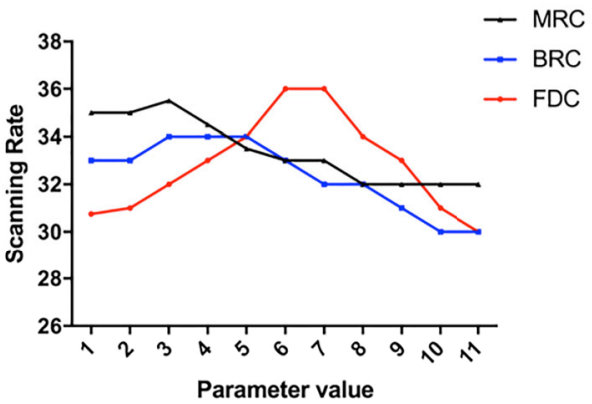

E

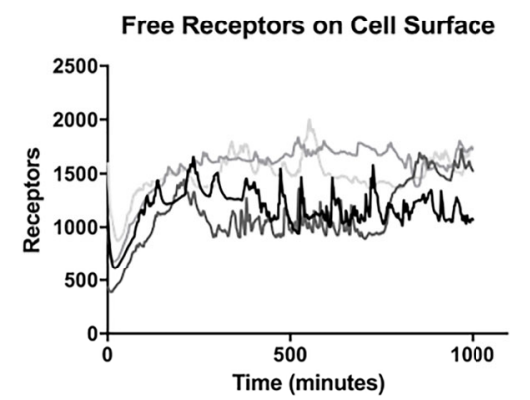

- Cell 1

- Cell 2

- Cell 3

- Cell 4
B

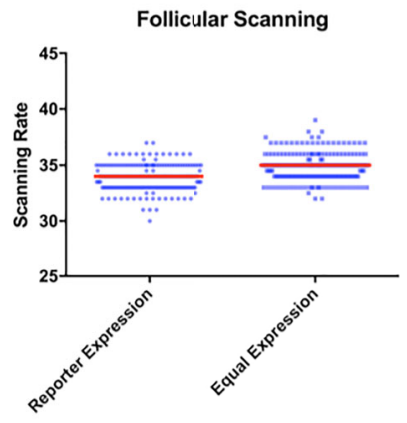

D

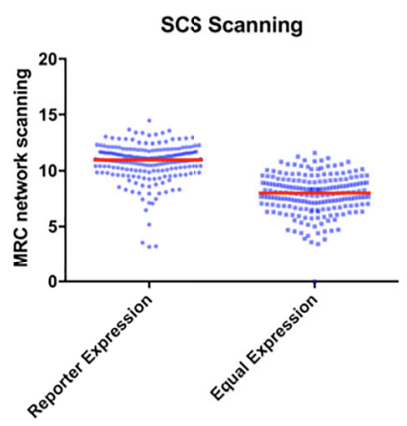

F
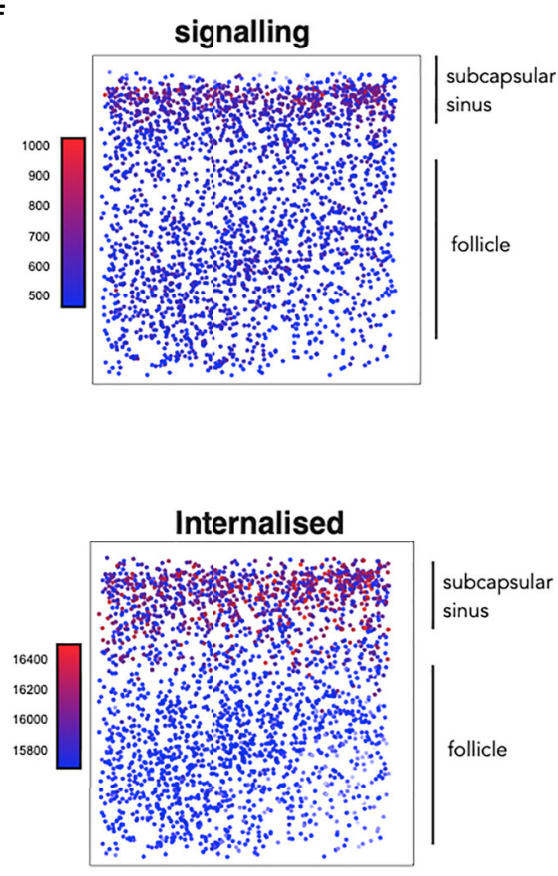

FIGURE 4 | Single cell tracking in silico to assess the spatiotemporal dynamics of CXCR5 expression and signaling. (A) Experimentally obtained in vivo CXCL13 reporter expression from different stromal cell subsets in the CXCL13-EYFP mouse. (B) In silico scanning rates for the entire follicle using equal secretion rates for all stromal cell subsets or secretion rates representative of CXCL13 reporter expression. (C) In silico scanning rates following OAT perturbations to secretion rates in different stromal cell subsets. (D) In silico scanning rates for the subcapsular sinus using equal secretion rates for all stromal cell subsets or secretion rates representative of CXCL13 reporter expression. (E) Single-cell tracking of CXCR5 expression on the cell surface. (Top) each line represents a distinct B cell within the same simulation run and (bottom) comparison of free and receptor signaling dynamics within a single cell. (F) Spatial dependence of CXCR5 signaling within the follicle. Each dot in the diagram represents the $X$ and $Y$ coordinates of a B cell agent in the simulator. The top of each square diagram is the subcapsular sinus. Each agent is colored by the number of receptors (as indicated by the title of each plot) with red representing high values and blue representing low values. 
mediated migration have highlighted a highly dynamic and intricate regulation network at the cell surface that allows immune cells to dynamically perceive the localized environment. Translating these findings in vivo is challenging due to the limitations of currently available experimental approaches. In this study we apply novel computational tools to address this limitation, and have explored the robustness of $\mathrm{B}$ cell scanning to perturbations in CXCR5 signaling and expression in silico.

Specifically, we extend upon previous models of chemotaxis in vitro, and in vivo models of lymph node stromal cells to better examine the migration of $\mathrm{B}$ cells within a complex tissue microenvironment. The use of ordinary differential equations within each agent permits single-cell analyses of CXCR5 expression with high spatiotemporal resolution. This key advantage allows the tool to serve as an adjunct to experimental approaches that are not capable of performing single cell analyses in situ. Flow cytometry approaches require cells to be isolated from a tissue, while confocal microscopy takes a static snapshot of a dynamic process. While single-cell sequencing is becoming more prevalent, linking this information back to spatial positioning within a tissue is challenging, with need for a robust panel of markers that differentiate cell subsets on the basis of spatial positioning or the use of micro-dissection. Similar issues arise when experimentally measuring CXCL13 within a tissue: fluorescent reporter systems cannot be used to tag the molecule itself, as the fluorescent label would drastically outweigh the molecule and alter its diffusive and binding characteristics. Our simulation platform may thus serve as a useful adjunct to further experimental studies of chemokine receptor activity, affording single-cell tracking precision within a 3-Dimensional complex environment beyond the capability of current imaging approaches.

Based on our quantification of parametric uncertainty, we find that while some kinetic parameters were unidentifiable, nonlinear interactions between kinetic parameters were driving simulation outputs, a finding consistent with a meta-analysis of parametric uncertainty in systems biology models (52). Importantly, this result was observed across many different parameter combinations and model iterations. In future work we aim to leverage this insight to simplify our model such that it can be generalized to other contexts.

Focusing on CXCL13-CXCR5 mediated regulation we find that in silico scanning of the $\mathrm{LN}$ follicle was robust to changes in total receptor numbers while complete loss of the receptor yielded a significant phenotype. This result is consistent with experimental studies showing that CXCR5 migration is also important in virally infected lymph nodes, where $\operatorname{cxcl13}$ gene expression is significantly reduced (8). This result, in conjunction with a suite of local and global sensitivity analyses led to the hypothesis that the dynamic modulation of CXCR5 signalling through rapid turnover and desensitization of receptors is a key determinant of antigen scanning. To explore our prediction, we used an MOEA approach to determine what combination of CXCR5 associated parameters gave rise to the highest scanning rates. Consistent with our hypothesis, this analysis supports a model whereby dynamic turnover of receptors and modulation of signalling are critical. Parameter distributions that gave rise to Pareto optimal solutions were skewed towards high rates of receptor ligand-binding, internalization, recycling, and desensitization rates.

To place our results in the context of the tissue microenvironment we also analyzed spatial and temporal aspects of CXCR5 activity. We assessed cell specific contributions to follicular scanning rates and show that FDCs are a key determinant of scanning within the primary lymph node follicle. Interestingly, using the fluorescent intensity values for each cell types to inform relative secretion rates yielded a CXCL13 landscape which promoted scanning at the subcapsular sinus, a site where large antigen enters the LN. This configuration may also promote shuttling of antigen by naïve $B$ cells to the FDC network for long-term storage. In addition, we performed single cell tracking experiments that predict that CXCR5 expression on the cell surface is dynamic, an emergent property that has been reported in other theoretical studies (14). Interestingly, CXCR5-mediated signalling was also spatially regulated - an emergent property where highest rates of signalling were observed at the SCS, close to the site of antigen entry into the lymph node parenchyma. Given our data, we hypothesize that dynamic modulation of receptors at the cell surface leads to fine-tuning of migratory responses within the Bcell niche. However, further analyses are required to determine the contribution of other chemotactic molecules and adhesion molecules to this phenomenon.

In further studies, we aim to extend this system by incorporating additional migratory factors such as CCL19/21, CXCL12 and 7,25 $\alpha$ hydroxycholesterol (1). The decision to omit these factors from the model was influenced by the lack of quantitative data for these molecules rather than a limitation of the computational platform. By incorporating these factors as more data becomes available we can begin assessing complex interactions between different receptors.

To summarize, our computational approach has enabled us to study the dynamics of chemokine receptor expression in the context of a complex tissue microenvironment. This work builds upon previous theoretical studies which study receptor dynamics in the context of idealized morphogen gradients where the complexity of tissue architecture is highly abstracted. Our data suggest that while receptor numbers are important in regulating migration they influence scanning rates to a lesser extent than the combined effect of receptor binding, desensitization, and internalization. The data also suggests that CXCR5 activity is modulated differently within different subfollicular niches. As such, our simulation platform is well placed to complement experimental work, given the relative speed at which data is acquired, the ability to perform single cell analyses, and the ability to assess how perturbations at one level of organization manifest at different scales.

\section{DATA AVAILABILITY STATEMENT}

The datasets presented in this study can be found in online repositories. The names of the repository/repositories and 
accession number(s) can be found below: https://www.kennedy. ox.ac.uk/technologies/resources/cxcl13sim.

\section{AUTHOR CONTRIBUTIONS}

JC designed and performed the experiments, analyzed, and interpreted the data and wrote the manuscript. KA and JS performed experiments, analyzed data, provided key reagents, intellectual input, and technical assistance. JT and MC designed experiments, analyzed and interpreted results, coordinated the research, and wrote the paper. All authors contributed to the article and approved the submitted version.

\section{FUNDING}

KA was supported by Wellcome Trust Centre for Future Health grant (204829), JT by EPSRC grant EP/K040820/1. JC, JT, and MC were funded by Wellcome Trust (Computational Approaches in Translational Science WT0905024MA, HFSP

\section{REFERENCES}

1. Pereira JP, Kelly LM, Cyster JG. Finding the Right Niche: B-Cell Migration in the Early Phases of T-Dependent Antibody Responses. Int Immunol (2010) 22 (6):413-9. doi: 10.1093/intimm/dxq047

2. Bennett LD, Fox JM, Signoret N. Mechanisms Regulating Chemokine Receptor Activity. Immunology (2011) 134(3):246-56. doi: 10.1111/j.13652567.2011.03485.x

3. Rot A, von Andrian UH. Chemokines in Innate and Adaptive Host Defense: Basic Chemokinese Grammar for Immune Cells. Annu Rev Immunol (2004) 22:891-928. doi: 10.1146/annurev.immunol.22.012703.104543

4. Förster R, Mattis AE, Kremmer E, Wolf E, Brem G, Lipp M. A Putative Chemokine Receptor, BLR1, Directs B Cell Migration to Defined Lymphoid Organs and Specific Anatomic Compartments of the Spleen. Cell (1996) 87 (6):1037-47. doi: 10.1016/S0092-8674(00)81798-5

5. Gunn MD, Ngo VN, Ansel KM, Ekland EH, Cyster JG, Williams LT. A BCell-Homing Chemokine Made in Lymphoid Follicles Activates Burkitt's Lymphoma Receptor-1. Nature (1998) 391(6669):799-803. doi: 10.1038/ 35876

6. Legler DF, Loetscher M, Roos RS, Clark-Lewis I, Baggiolini M, Moser B. B Cell-Attracting Chemokine 1, A Human CXC Chemokine Expressed in Lymphoid Tissues, Selectively Attracts B Lymphocytes via BLR1/CXCR5. J Exp Med (1998) 187(4):655-60. doi: 10.1084/jem.187.4.655

7. Ansel KM, Ngo VN, Hyman PL, Luther SA, Förster R, Sedgwick JD, et al. A Chemokine-Driven Positive Feedback Loop Organizes Lymphoid Follicles. Nature (2000) 406(6793):309-14. doi: 10.1038/35018581

8. Coelho FM, Natale D, Soriano SF, Hons M, Swoger J, Mayer J, et al. Naive BCell Trafficking is Shaped by Local Chemokine Availability and LFA-1Independent Stromal Interactions. Blood (2013) 121(20):4101-9. doi: 10.1182/blood-2012-10-465336

9. Herzmark P, Campbell K, Wang F, Wong K, El-Samad H, Groisman A, et al. Bound Attractant at the Leading vs. The Trailing Edge Determines Chemotactic Prowess. Proc Natl Acad Sci USA (2007) 104(33):13349-54. doi: 10.1073/pnas.0705889104

10. Zigmond SH. Consequences of Chemotactic Peptide Receptor Modulation for Leukocyte Orientation. J Cell Biol (1981) 88(3):644-7. doi: 10.1083/ jcb.88.3.644

11. Sh Z, Sj S, Da L. Kinetic Analysis of Chemotactic Peptide Receptor Modulation., Kinetic Analysis of Chemotactic Peptide Receptor Modulation. J Cell Biol (1982) 92(1, 1):34, 34-43. doi: 10.1083/jcb.92.1.34
(RGP0006/2009 MC) and Medical Research Grants MR/ K021125/1 and G0601156. MC is funded by the Kennedy Trust.

\section{ACKNOWLEDGMENTS}

We wish to thank Antal Rot, Paul Kaye, Dimitris Lagos and members of the Hull/York Medical School for advice and reagents, the York Teaching Hospital NHS Foundation Trust R\&D Department for invaluable assistance with sample collection protocol and Imaging \& Cytometry Laboratory staff for technical input. The content of this manuscript has been published as part of the thesis of JC (53).

\section{SUPPLEMENTARY MATERIAL}

The Supplementary Material for this article can be found online at: https://www.frontiersin.org/articles/10.3389/fimmu.2021. 703088/full\#supplementary-material

12. Aronin CEP, Zhao YM, Yoon JS, Morgan NY, Prüstel T, Germain RN, et al. Migrating Myeloid Cells Sense Temporal Dynamics of Chemoattractant Concentrations. Immunity (2017) 47(5):862-74.e3. doi: 10.1016/ j.immuni.2017.10.020

13. Calebiro D, Rieken F, Wagner J, Sungkaworn T, Zabel U, Borzi A, et al. SingleMolecule Analysis of Fluorescently Labeled G-Protein-Coupled Receptors Reveals Complexes With Distinct Dynamics and Organization. Proc Natl Acad Sci USA (2013) 110(2):743-8. doi: 10.1073/pnas.1205798110

14. Chan C, Billard M, Ramirez SA, Schmidl H, Monson E, Kepler TB. A Model for Migratory B Cell Oscillations From Receptor Down-Regulation Induced by External Chemokine Fields. Bull Math Biol (2013) 75(1):185-205. doi: 10.1007/s11538-012-9799-9

15. Beyer T, Meyer-Hermann M. Cell Transmembrane Receptors Determine Tissue Pattern Stability. Phys Rev Lett (2008) 101(14):148102. doi: 10.1103/ PhysRevLett.101.148102

16. Figge MT, Garin A, Gunzer M, Kosco-Vilbois M, Toellner K-M, MEYERHERMANN M. Deriving a Germinal Center Lymphocyte Migration Model From Two-Photon Data. J Exp Med (2008) 205(13):3019-29. doi: 10.1084/ jem. 20081160

17. Cosgrove J, Novkovic M, Albrecht S, Pikor NB, Zhou Z, Onder L, et al. B Cell Zone Reticular Cell Microenvironments Shape CXCL13 Gradient Formation. Nat Commun (2020) 11(1):3677. doi: 10.1038/s41467-020-17135-2

18. Andrews P, Timmis J, Polack F, Stepney S, Sampson A. The CoSMoS Process, Version 0.1: A Process for the Modelling and Simulation of Complex Systems. Technical Report. YCS-2010-453. York, United Kingdom: University of York (2010).

19. Alden K, Andrews PS, Polack FAC, Veiga-Fernandes H, Coles MC, Timmis J. Using Argument Notation to Engineer Biological Simulations With Increased Confidence. J R Soc Interface (2015) 12(104):20141059. doi: 10.1098/ rsif.2014.1059

20. Cosgrove J, Butler J, Alden K, Read M, Kumar V, Cucurull-Sanchez L, et al. Agent-Based Modeling in Systems Pharmacology. CPT Pharmacometrics Syst Pharmacol (2015) 4(11):615-2. doi: 10.1002/psp4.12018

21. Luke S, Cioffi-Revilla C, Panait L, Sullivan K, Balan G. MASON: A Multiagent Simulation Environment. Simulation (2005) 81(7):517-27. doi: 10.1177/ 0037549705058073

22. Sommerville I. Software Engineering. 9 edition Vol. 792. Boston: Pearson (2010).

23. Irla M, Guenot J, Sealy G, Reith W, Imhof BA, Sergé A. Three-Dimensional Visualization of the Mouse Thymus Organization in Health and 
Immunodeficiency. J Immunol (Baltimore Md: 1950) (2013) 190(2):586-96. doi: $10.4049 /$ jimmunol.1200119

24. Varsha K, Scandella E, Danuser R, Onder L, Nitschké M, Fukui Y, et al. Global Lymphoid Tissue Remodeling during a Viral Infection Is Orchestrated by a B Cell-Lymphotoxin-Dependent Pathway. Blood (2010) 115(23):4725-33. doi: 10.1182/blood-2009-10-250118

25. Monroe JG, Cambier JC. Sorting of B Lymphoblasts Based Upon Cell Diameter Provides Cell Populations Enriched in Different Stages of Cell Cycle. J Immunol Methods (1983) 63(1):45-56. doi: 10.1016/0022-1759(83)90208-9

26. Barroso R, Martínez Muñoz L, Barrondo S, Vega B, Holgado BL, Lucas P, et al. EBI2 Regulates CXCL13-Mediated Responses by Heterodimerization With CXCR5. FASEB J (2012) 26(12):4841-54. doi: 10.1096/fj.12-208876

27. Tilo B, M-H M. Modeling Emergent Tissue Organization Involving High-Speed Migrating Cells in a Flow Equilibrium. Phys Rev E Stat Nonlin Soft Matter Phys (2007) 76(2 Pt 1):021929. doi: 10.1103/PhysRevE.76.021929

28. Luther SA, Bidgol A, Hargreaves DC, Schmidt A, Xu Y, Paniyadi J, et al. Differing Activities of Homeostatic Chemokines CCL19, CCL21, and CXCL12 in Lymphocyte and Dendritic Cell Recruitment and Lymphoid Neogenesis. J Immunol (2002) 169(1):424-33. doi: 10.4049/jimmunol.169.1.424

29. Wang Y, Irvine DJ. Convolution of Chemoattractant Secretion Rate, Source Density, and Receptor Desensitization Direct Diverse Migration Patterns in Leukocytes. Integr Biol (Camb) (2013) 5(3):481-94. doi: 10.1039/c3ib20249f

30. Phair RD, Misteli T. Kinetic Modelling Approaches to In Vivo Imaging. Nat Rev Mol Cell Biol (2001) 2(12):898-907. doi: 10.1038/35103000

31. Sklar LA, Finney DA, Oades ZG, Jesaitis AJ, Painter RG, Cochrane CG. The Dynamics of Ligand-Receptor Interactions. Real-Time Analyses of Association, Dissociation, and Internalization of an N-Formyl Peptide and Its Receptors on the Human Neutrophil. J Biol Chem (1984) 259(9):5661-69.

32. Beyer T, Meyer-Hermann M. Modeling Emergent Tissue Organization Involving High-Speed Migrating Cells in a Flow Equilibrium. Phys Rev E Stat Nonlinear Soft Matter Phys (2007) 76(2 Pt 1):021929. doi: 10.1103/PhysRevE.76.021929

33. Luther SA, Bidgol A, Hargreaves DC, Schmidt A, Xu Y, Paniyadi J, et al. Differing Activities of Homeostatic Chemokines CCL19, CCL21, and CXCL12 in Lymphocyte and Dendritic Cell Recruitment and Lymphoid Neogenesis. J Immunol (2002) 169:424-33. doi: 10.4049/jimmunol.169.1.424

34. Barroso R, Muñoz LM, Barrondo S, Vega B, Holgado BL, Lucas P, et al. EBI2 Regulates CXCL13-Mediated Responses by Heterodimerization with CXCR5. FASEB J: Off Publ Fed Am Soc Exp Biol (2012) 26(12):4841-54. doi: 10.1096/ fj.12-208876

35. Kohout TA, Nicholas SL, Perry SJ, Reinhart G, Junger S, Struthers RS. Differential Desensitization, Receptor Phosphorylation, Beta-Arrestin Recruitment, and ERK1/2 Activation by the Two Endogenous Ligands for the CC Chemokine Receptor 7. J Biol Chem (2004) 279(22):23214-22. doi: $10.1074 /$ jbc.M402125200

36. Otero C, Groettrup M, Legler DF. Opposite Fate of Endocytosed CCR7 and Its Ligands: Recycling versus Degradation. J Immunol (Baltimore Md: 1950) (2006) 177(4):2314-23.

37. Schwarz J. A Microfluidic Device for Measuring Cell Migration towards Substrate-Bound and Soluble Chemokine Gradients. Sci Rep (2016) 6:36440.

38. Eran E, Geva-Zatorsky N, Issaeva I, Cohen A, Dekel E, Danon T, et al. Proteome Half-Life Dynamics in Living Human Cells. Sci (New York NY) (2011) 331:764-68. doi: 10.1126/science.1199784

39. Schwanhäusser B, Busse D, Li N, Dittmar G, Schuchhardt J, Wolf J, et al. Global Quantification of Mammalian Gene Expression Control. Nature (2011) 473:337-42. doi: 10.1038/nature10098

40. Milo R, Phillips R. Cell Biology by the Numbers. 1 edition. New York, NY: Garland Science (2015).
41. Royston P. Approximating the Shapiro-Wilk W-Test for non-Normality. Stat Comput (1992) 2(3):117-9. doi: 10.1007/BF01891203

42. Alden K, Read M, Timmis J, Andrews PS, Veiga-Fernandes H, Coles M. Spartan: A Comprehensive Tool for Understanding Uncertainty in Simulations of Biological Systems. PloS Comput Biol (2013) 9(2):e1002916: 10.1371/journal.pcbi.1002916.

43. Vargha A, Delaney HD. A Critique and Improvement of the 'CL' Common Language Effect Size Statistics of McGraw and Wong. J Educ Behav Stat (2000) 25(2):101-32. doi: 10.2307/1165329

44. Read MN. Statistical and Modelling Techniques to Build Confidence in the Investigation of Immunology Through Agent-Based Simulation. University of York (2011). Available at: http://etheses.whiterose.ac.uk/2174/.

45. Vargha A, Delaney HD. A Critique and Improvement of the "Cl" Common Language Effect Size Statistics of McGraw and Wong. J Educ Behav Statistics (2000) 25(2):101-32. doi: 10.2307/1165329

46. Marino S, Hogue IB, Ray CJ, Kirschner DE. A Methodology for Performing Global Uncertainty and Sensitivity Analysis in Systems Biology. J Theor Biol (2008) 254(1):178-96. doi: 10.1016/j.jtbi.2008.04.011

47. Saltelli A, Bolado R. An Alternative Way to Compute Fourier Amplitude Sensitivity Test (FAST). Comput Stat Data Anal (1998) 26(4):445-60. doi: 10.1016/S0167-9473(97)00043-1

48. Saltelli A, Chan K, Scott EM. Sensitivity Analysis. 1 edition Vol. 494. New York: Chichester; Weinheim etc: Wiley-Blackwell (2008).

49. Deb K, Pratap A, Agarwal S, Meyarivan T. A Fast and Elitist Multiobjective Genetic Algorithm: NSGA-II. IEEE Trans Evolutionary Computation (2002) 6 (2):182-97. doi: 10.1109/4235.996017

50. Lin F, Butcher EC. Modeling the Role of Homologous Receptor Desensitization in Cell Gradient Sensing. J Immunol (2008) 181(12):833543. doi: $10.4049 /$ jimmunol.181.12.8335

51. Onder L, Mörbe U, Pikor N, Novkovic M, Cheng H-W, Hehlgans T, et al. Lymphatic Endothelial Cells Control Initiation of Lymph Node Organogenesis. Immunity (2017) 47(1):80-92.e4. doi: 10.1016/ j.immuni.2017.05.008

52. Gutenkunst RN, Waterfall JJ, Casey FP, Brown KS, Myers CR, Sethna JP. Universally Sloppy Parameter Sensitivities in Systems Biology Models. PloS Comput Biol (2007) 3(10). doi: 10.1371/journal.pcbi.0030189

53. Cosgrove J. The Spatial Distribution and Dynamics of CXCL13 in Lymphoid Tissues. York, United Kingdom: University of York (2017). p. e189. Available at: http:// etheses.whiterose.ac.uk/19258/.

Conflict of Interest: The authors declare that the research was conducted in the absence of any commercial or financial relationships that could be construed as a potential conflict of interest.

Publisher's Note: All claims expressed in this article are solely those of the authors and do not necessarily represent those of their affiliated organizations, or those of the publisher, the editors and the reviewers. Any product that may be evaluated in this article, or claim that may be made by its manufacturer, is not guaranteed or endorsed by the publisher.

Copyright $\odot 2021$ Cosgrove, Alden, Stein, Coles and Timmis. This is an open-access article distributed under the terms of the Creative Commons Attribution License (CC BY). The use, distribution or reproduction in other forums is permitted, provided the original author(s) and the copyright owner(s) are credited and that the original publication in this journal is cited, in accordance with accepted academic practice. No use, distribution or reproduction is permitted which does not comply with these terms. 